\author{
L. Baniadam ${ }^{1}$, S. Arshi 1 , M. Nabavi ${ }^{1}$, A. Yousefr ${ }^{2}$, M. Fallahpour ${ }^{1}$, S. Shokri ${ }^{1}$, \\ F. Zare MehrJerdi ${ }^{1}$, F. Salari ${ }^{1}$, M. H. Bemanian ${ }^{1}$
}

\title{
Can concurrent lower gastrointestinal manifestations help the timely diagnosis of small intestinal bacterial overgrowth in CVID patients?
}

\begin{abstract}
${ }^{1}$ Department of Allergy and Clinical Immunology, Rasool e Akram Hospital, Iran University of Medical Sciences, Tehran, Iran

${ }^{2}$ Department of Pediatric Gastroenterology, Rasool e Akram Hospital, Iran University of Medical Sciences, Tehran, Iran
\end{abstract}

\section{KEY WORDS}

Common Variable Immunodeficiency (CVID); Small Intestinal Bacterial

Overgrowth (SIBO); chronic diarrhea;

bydrogen breath test; primary

immunodeficiency; gastrointestinal

symptoms.

\section{Corresponding author}

Mohammad Hassan Bemanian

Department of Allergy and Clinical

Immunology

Rasool e Akram Hospital

Iran University of Medical Sciences

Niayesh Street, Satarkhan Avenue

Tehran 1449614535, Islamic Republic

of Iran

E-mail: mhbemanian@yahoo.com

Doi

10.23822/EurAnnACI.1764-1489.137

\begin{abstract}
Summary
Introduction and objective. Gastrointestinal complications are considered as one of the most common manifestations in patients with Common Variable Immunodeficiency (CVID). These complications can result from Small Intestinal Bacterial Overgrowth (SIBO). Hydrogen breath test is extensively used to diagnose SIBO. The objective of this study was to evaluate the prevalence of SIBO using the Hydrogen Breath Test (HBT) in patients with CVID. Materials and methods. Twenty-seven patients with CVID entered this cross-sectional study. Demographic and lower gastrointestinal symptoms were recorded in a check list. Hemoglobin level was measured in all patients. The concentration of IgA and IgG was assessed using nephelometry. Moreover, SIBO was detected by means of Glucose hydrogen breath test. Results. The mean $( \pm S D)$ age of the patients was $35.25( \pm 11.69)$ years. Twenty patients $(74.1 \%)$ manifested at least one lower gastrointestinal symptom. The most frequent lower gastrointestinal manifestations were bloating (66.7\%) and chronic diarrhea (40.7\%), respectively. IgA level less than $10 \mathrm{mg} / \mathrm{dl}$ and IgG level less than $600 \mathrm{mg} / \mathrm{dl}$ were determined in $77.8 \%$ and $25.9 \%$ of patients, respectively. Positive HBT was detected in $40.7 \%(n=11)$ of the patients. In the positive HBT group, bloating, chronic diarrhea and abdominal pain were the most common lower GI manifestations. There was no significant difference in terms of age, BMI, IgA level, and duration of CVID between the positive and negative HBT groups. The significant association of co-occurrence of anemia and abdominal pain with positive HBT (positive predictive value: 100\%) might be considered as a clue to SIBO diagnosis. Conclusions. Regarding the high prevalence and non-specific manifestation of SIBO, it is suggested to consider concurrent symptoms in patients with CVID to manage the timely and precise diagnosis of SIBO.
\end{abstract}

\section{Introduction}

Primary Immunodeficiency Diseases (PIDs) as a group of heterogeneous disorders are characterized by inability of the immune system to protect the body against foreign hazards $(1,2)$. Gastrointestinal (GI) problems are ranked as the second common manifestations in PID patients (2). It is noteworthy that GI complications are variable in different PIDs as well as different individuals (2).
Common Variable Immunodeficiency (CVID) as a heterogeneous Primary Immunodeficiency Disease (PID) with a prevalence of 1 in 25,000 to 50,000 is characterized by hypogammaglobulinemia, failure to produce immunoglobulin against vaccines and recurrent bacterial infections (3-5). Increased susceptibility to bacterial infections in respiratory and gastrointestinal tracts has been reported in more than $90 \%$ of patients with CVID (6). Given that the gastrointestinal tract contains a lymphatic system, it can be involved up to $50 \%$ of primary immunodeficiency diseases (5). Gastrointestinal manifes- 
tations are infections, inflammation, autoimmunity, and cancers (5). These gastrointestinal symptoms have different pathogenesis and do not often improve following conventional treatments (5). Previous studies have reported acute or chronic diarrhea as the most frequent gastrointestinal manifestations ranged from 20 to $60 \%$ in patients with CVID (7-9). The causes of chronic diarrhea in these patients are microbial and non-microbial. One of the major causes of diarrhea in these patients is small intestinal bacterial overgrowth that can be managed by broad-spectrum antibiotics $(10,11)$.

Although Small Intestinal Bacterial Overgrowth (SIBO) is common in patients with CVID, its diagnosis is difficult owing to continuous or interrupted use of antibiotics (11). The small intestine aspirate and its culture as well as Hydrogen Breath Test (HBT) are applied as two current clinical tests to detect SIBO. Indeed, HBT is a cheap, easy to use and non-invasive test. Moreover it is regarded as the most popular test to diagnose SIBO in high-risk patients (12). The aim of this study was to assess the prevalence of SIBO as one of the reasons for chronic diarrhea in patients with CVID.

\section{Materials and methods}

This cross-sectional study was conducted in Rasool Akram Hospital from 2009 to 2019. All patients with CVID enrolled in the study, according to the diagnostic criteria of ESID 2014 (13). This study was approved by the Ethics Committee of Iran University of Medical Sciences (N: IUMS.FMD.REC.1397.177). The inclusion criterion required was the correct preparation prior to perform a hydrogen breath test.

All characteristics including demographic data and lower gastrointestinal symptoms (chronic diarrhea, bloating, abdominal pain, constipation and steatorrhea) were recorded for all patients. The concentration of hemoglobin was assessed. Furthermore, the level of $\operatorname{IgA}$ and $\operatorname{IgG}$ was measured using nephelometry (Eppendorf, Ecom-EG125 system, Germany) during Ig replacement therapy and before receiving the last dose of IVIG. To assess SIBO, HBT was performed using Fischer system (Lacto FAN2, Germany).

\section{Hydrogen Breath Test (HBT)}

To do this test, patients were asked to consider some precautions including (1) not taking antibiotics for 4 weeks prior to the test, (2) avoidance of carbohydrates foods (such as bread, potato and spaghetti) for $24 \mathrm{~h}$ before the test, (3) not taking any fiber supplements and laxatives $24 \mathrm{~h}$ before the test, (4) fasting for 8-12 h before the test, (5) avoidance of smoking, sleeping and physical activity 30 minutes before and during the test (6) and the use of chlorhexidine mouthwash before the test.

The baseline of breath hydrogen was evaluated for all patients. Then, $75 \mathrm{~g}$ of glucose dissolved in the water was ingested by patients. The HBT was repeated every 20 minutes for 120 minutes. An increase of 20 part per million (ppm) in hydrogen compared to baseline at least two times during 90 minutes was considered to be a positive test (12).

\section{Statistical analysis}

To analyze the data, a set of quantitative and qualitative statistical tests available in the SPSS software version 20 (IBM, Armonk, NY, USA) was used. To describe the categorical variables, the frequency and percent were determined. Mean \pm SD and median $\left(25^{\text {th }}\right.$ percentile $(\mathrm{Q} 1)$, $75^{\text {th }}$ percentile $(\mathrm{Q} 3)$ ) were calculated to describe the normal and non-normal quantitative variables, respectively. In order to compare the mean difference of a quantitative variable between two dependent groups, Independent t-test or Mann-Whitney test were applied. To evaluate the relationship between two categorical variables, Chi Square or Fisher Exact test were used. The significant level was considered less than 0.05. Graphpad Prism 5 (Graphpad Software Inc., La Jolla, CA, USA) software was applied for drawing the graphs.

\section{Results}

The mean $( \pm$ SD) age of the 27 included patients was $35.25( \pm 11.69)$ years. Sixteen subjects $(59.3 \%)$ were female. The mean $( \pm S D)$ of Body Mass Index (BMI) was $22.27 \pm 3.89$. The median (Q1, Q3) duration of CVID was $9(4,14)$ year. Seven patients $(25.9 \%)$ had a BMI less than 20. Anemia was detected in 33.3\% $(n=9)$ of the patients. Among 9 patients with anemia, four and five patients had microcytic anemia and normocytic anemia, respectively. In addition, $77.8 \%$ and $25.9 \%$ of the patients with CVID showed IgA level less than $10 \mathrm{mg} / \mathrm{dl}$ and IgG level less than $600 \mathrm{mg} / \mathrm{dl}$, respectively. Twenty patients (74.1\%) presented at least one lower gastrointestinal symptom. The frequency of lower gastrointestinal manifestations is shown in figure $\mathbf{1}$. As the figure demonstrates, the most frequent lower gastrointestinal symptoms were bloating $(66.7 \%)$ and chronic diarrhea (40.7\%), respectively.

Table I shows findings of GI symptoms, clues for malabsorption (anemia, BMI), immunoglobulin deficiency (IgG, IgA) and GI comorbidities in the positive and negative HBT group. Hydrogen Breath Test $(\mathrm{HBT})$ was positive in $40.7 \%(\mathrm{n}=11)$ of the patients. In the CVID patients with positive HBT, bloating, chronic diarrhea and abdominal pain were the most common lower GI manifestations. No significant difference was found in terms of age, BMI, IgA, IgG and duration of CVID according to the result of HBT. The gastrointestinal comorbidities such as Inflammatory Bowel Diseases (IBD) and polyposis of the ileum were reported through records of patients. Among the studied patients, two IBD cases with positive HBT, two cases with polyposis of the ileum (one positive HBT) and two cases with Nodular Lymphoid Hyperplasia (NLH) (one positive HBT) were reported.

SomeSIBO-associated symptoms such as abdominal pain and bloating were higher in HBT-positive group compared to HBT-negative group. A significant relationship was found between co-occurrence 
Figure 1 - The frequency and percent of lower gastrointestinal manifestations in patients with CVID.

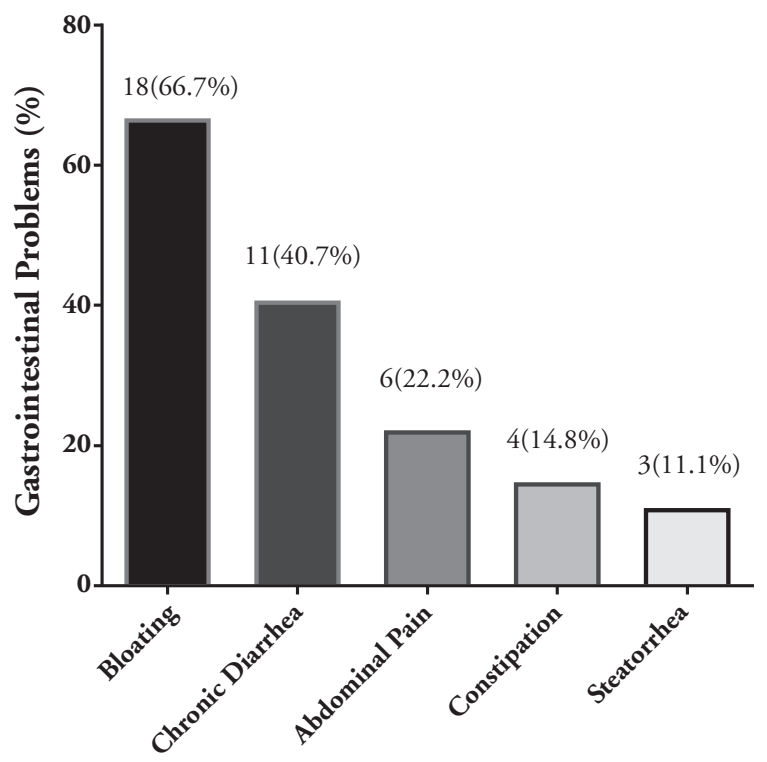

Table I - The characteristics of CVID patients with positive and negative HBT results.

\begin{tabular}{|c|c|c|c|c|c|}
\hline & & Total & Positive HBT & Negative HBT & P value \\
\hline \multirow{4}{*}{$\begin{array}{l}\text { Gastrointestinal Symptoms } \\
\text { N (\%) }\end{array}$} & Bloating & $18(66.7)$ & $8(72.7)$ & $10(62.5)$ & 0.69 \\
\hline & Chronic diarrhea & $11(40.7)$ & $6(54.5)$ & $5(31.3)$ & 0.26 \\
\hline & Constipation & $4(14.8)$ & $1(9.1)$ & $3(18.8)$ & 0.62 \\
\hline & Steatorrhea & $3(11.1)$ & $2(18.2)$ & $1(6.3)$ & 0.54 \\
\hline $\begin{array}{l}\text { Clues for malabsorption } \\
\text { N (\%) }\end{array}$ & $\mathrm{BMI}<20$ & $7(25.9)$ & $3(27.3)$ & $4(25)$ & 1.00 \\
\hline Immunoglobulin deficiency & $\begin{array}{l}\mathrm{IgG}(\mathrm{mg} / \mathrm{dl}) \\
\text { Median }\end{array}$ & $714(500,770)$ & $760(460,830)$ & $700(525,736)$ & 0.29 \\
\hline Median (Q1, Q3) & $\mathrm{IgG}<600 \mathrm{mg} / \mathrm{dl}$ & $7(25.9)$ & $3(27.3)$ & $4(25)$ & 1.00 \\
\hline \multicolumn{6}{|l|}{ N (\%) } \\
\hline & $\operatorname{IgA}<10 \mathrm{mg} / \mathrm{dl}$ & $21(77.8)$ & $9(81.8)$ & $12(75)$ & 1.00 \\
\hline \multirow[t]{3}{*}{$\begin{array}{l}\text { GI comorbidities } \\
\text { N }(\%)\end{array}$} & $\begin{array}{l}\text { Inflammatory Bowel } \\
\text { Diseases (IBD) }\end{array}$ & $2(7.4)$ & $2(18.2)$ & $0(0)$ & 0.15 \\
\hline & Polyposis of the ileum & $2(7.4)$ & $1(9.1)$ & $1(6.3)$ & 1.00 \\
\hline & $\begin{array}{l}\text { Nodular lymphoid } \\
\text { hyperplasia }\end{array}$ & $2(7.4)$ & $1(9.1)$ & $1(6.3)$ & 1.00 \\
\hline
\end{tabular}


of anemia and abdominal pain with positive HBT $(\mathrm{P}=0.05)($ table II). A positive predictive value of $100 \%$ was achieved for anemia accompanied with abdominal pain. Taking into account the small number of studied cases and the statistical results, which do not reach significance, it would be better to more cautiously state that HBT-positive patients tend to be those patients with IgG less than $600 \mathrm{mg} / \mathrm{dl}$ and abdominal pain. Other combinations (abdominal pain + bloating, anemia + bloating, chronic diarrhea and $\operatorname{IgA}<10$ $\mathrm{mg} / \mathrm{dl}$, abdominal pain and $\mathrm{IgA}<10 \mathrm{mg} / \mathrm{dl}$ ) seem to be less specific, although greater numbers are associated with HBT positivity.

\section{Discussion}

Herein, we assessed the prevalence of Small Intestinal Bacterial Overgrowth (SIBO) by using the Hydrogen Breath Test (HBT) in patients with CVID. As the results show, bloating and chronic diarrhea were found as the most common lower gastrointestinal symptoms in patients with CVID. Moreover, SIBO was detected in more than $40 \%$ of the patients.

As the literature review shows, the frequency of SIBO has been underestimated in the last decades, resulting in misdiagnosis and remarkable clinical complications in patients $(12,14)$. There were some reasons for explaining this issue, including a wide spectrum of SIBO-related non-specific clinical symptoms, lack of consensus guidelines for diagnosis as well as a gold standard test to precisely to detect the frequency of SIBO. These issues could make the diagnosis and treatment more complicated and challenging. In other words, the accurate prevalence of SIBO and its association with a specific disease is unclear (12) . Although SIBO is one of the main and common causes of chronic diarrhea in patients with CIVD, it is difficult to be diagnosed because of constant or irregular antibiotic usage in these patients (11).

In the current study conducted on patients with CVID, no significant relationship was found between the frequency of SIBO and age, gender, and duration of CVID. According to the literature, a relationship was found between the prevalence of SIBO and age and gender (15).

In this study, lower gastrointestinal manifestations were observed in $74.1 \%$ of the patients and bloating and chronic diarrhea were the two most frequently reported lower GI symptoms. In line with our findings, the small intestinal bacterial overgrowth often leads to bloating and chronic diarrhea (14). Moreover, in HBT-positive group, $81 \%$ of patients manifested symptoms associated with SIBO, while the others $(19 \%)$ manifested none. Given that the symptoms related to SIBO vary in severity from asymptomatic to severe, it can be presumed that in asymptomatic cases, strains grown do not affect the gastrointestinal mucosa (12).

Chronic diarrhea was seen in $40.7 \%$ of our patients. Hermanszewsk $e$ t al. reported a similar frequency $(40 \%)$ of chronic diarrhea in patients with CVID (16). On the other hand, a higher prevalence of chronic diarrhea (up to 60\%) was found in studies by Hemans et al. (17) and Atarod et al. (18). In HBT-positive cases, bloating was reported as the most common GI manifestation. In line with this study, Mattsson $e t$ al. performed breath test in a population of adult patients, who indicated bloating as the most common symptom. As their study demonstrated, $46 \%$ of patients showed positive breath test (19).

In severe cases, patients with SIBO may develop severe weight loss as a result of diarrhea, insufficient food intake, and most notably nutrient malabsorption. Deficiency of vitamins, such as $B 12$ results in anemia in these patients $(10,20)$. As the present study revealed, microcytic or normocytic anemia was detected in $33.3 \%$ and $45.5 \%$ of CVID patients accompanying with positive HBT. Although Vitamin B12 deficiency may result in a megaloblastic anemia in severe cases, iron deficiency has also been described in SIBO but the mechanisms need further elucidation (12). On the other hand, our cases have a chronic disease, and they may have anemia because of it.

Table II - The relationship between co-occurrence of clinical symptoms and Small Intestinal Bacterial Overgrowth (SIBO) in patients with CVID results.

\begin{tabular}{|c|c|c|c|c|}
\hline & $\begin{array}{l}\text { Total } \\
\text { N }(\%)(95 \% \text { CI })\end{array}$ & $\begin{array}{l}\text { Positive HBT } \\
\text { N }(\%)\end{array}$ & $\begin{array}{l}\text { Negative HBT } \\
\text { N (\%) }\end{array}$ & P value \\
\hline Abdominal Pain and Anemia & $3(11.1)(5.06,17.14)$ & $3(100)$ & $0(0)$ & 0.05 \\
\hline Anemia and Bloating & $6(22.2)(6.53,37.87)$ & $4(66.7)$ & $2(33.3)$ & 0.18 \\
\hline Chronic Diarrhea and IgA $<10$ & $9(33.3)(15.6,51)$ & $5(55.6)$ & $4(44.4)$ & 0.41 \\
\hline Abdominal pain and $\operatorname{IgA}<10$ & $4(14.8)(1.5,28.1)$ & $3(75)$ & $1(25)$ & 0.27 \\
\hline Abdominal pain and IgG $<600$ & $2(7.4)(-2.47,17.27)$ & $2(100)$ & $0(0)$ & 0.15 \\
\hline
\end{tabular}

CVID (Common Variable Immunodeficiency); HBT (Hydrogen Breath Test). 
There is a positive relationship between anemia and other symptoms associated with SIBO since the co-occurrence of anemia with abdominal pain was significantly higher in patients with SIBO. Positive predictive value of $100 \%$ was obtained for anemia accompanied by abdominal pain, which can provide a clue to SIBO diagnosis. A higher incidence of SIBO in patients with abdominal pain + bloating, anemia + bloating, abdominal pain + $\operatorname{IgG}<600$, IgA less than $10 \mathrm{mg} / \mathrm{dl}+$ diarrhea as well as IgA less than $10 \mathrm{mg} / \mathrm{dl}$ and abdominal pain was observed. This finding is consistent with previous studies suggesting that GI infections are more common in patients with undetectable serum IgA level (21).

In this study, two limitations need to be considered. Firstly, the frequent use of antibiotics in patients with CVID owing to underlying diseases decreased the number of the patients able to perform the HBT, thereby leading to small sample size. Secondly, the exclusion of some patients due to respiratory problems and inability to perform HBT, reduced the number of the patients. Moreover, cross-sectional design of the study might also underestimate the number of patients with SIBO.

\section{Conclusions}

Regarding the findings of the current cross-sectional study, bloating and chronic diarrhea were considered as the most common lower gastrointestinal symptoms in patients with CVID and SIBO was detected in more than $40 \%$ of these patients. Moreover, a $100 \%$ positive predictive value for anemia accompanied with by abdominal pain. Notwithstanding the limitations due to the small number of cases, gastrointestinal symptoms and routine clinical evaluation arise as potential valuable diagnostic clues. Timely and precise diagnosis of SIBO may lead to appropriate management of manifestations using broad-spectrum antibiotics and thereby preventing weight loss and malabsorption stemmed from diarrhea and finally improve the quality of life in these patients.

\section{Fundings}

This study was supported by Iran University of Medical Sciences.

\section{Conflict of interests}

The authors declare that they have no conflict of interests.

\section{References}

1. McCusker C, Upton J, Warrington R. Primary immunodeficiency. Allergy Asthma Clin Immunol 2018;14(Suppl):61.

2. Schwimmer D, Glover S. Primary Immunodeficiency and the Gut. Gastroenterol Clin North Am 2019;48(2):199-220.

3. Mandaliya R, Burkart AL, DiMarino AJ, Rattan S, Cohen S. Association between common variable immunodeficiency and collage- nous infiltrative disorders of the gastrointestinal tract: A series of four patients. Indian J Gastroenterol 2016;35(2):133-8.

4. Ramirez-Vargas N, Arablin-Oropeza SE, Mojica-Martinez D, et al. Clinical and immunological features of common variable immunodeficiency in Mexican patients. Allergol Immunopathol 2014; $42(3): 235-40$.

5. Agarwal S, Mayer L. Diagnosis and treatment of gastrointestinal disorders in patients with primary immunodeficiency. Clin Gas troenterol Hepatol 2013;11(9):1050-63.

6. Salzer U, Warnatz K, Peter HH. Common variable immunodefi ciency: an update. Arthritis Res Ther 2012;14:223.

7. Cunningham-Rundles C. The many faces of common variable immunodeficiency. Hematology Am Soc Hematol Educ Program 2012;2012:301-5.

8. Maarschalk-Ellerbroek L. Clinical aspects of Common Variable Immunodeficiency: There is more to the image than meets the eye. University Utrecht 2013.

9. Uzzan M, Ko HM, Mehandru S, Cunningham-Rundles C. Gastrointestinal Disorders Associated with Common Variable Immune Deficiency (CVID) and Chronic Granulomatous Disease (CGD). Curr Gastroenterol Rep 2016;18(4):17.

10. Dukowicz AC, Lacy BE, Levine GM. Small intestinal bacterial overgrowth: a comprehensive review. Gastroenterol Hepatol (NY) 2007;3(2):112-22.

11. Agarwal S, Cunningham-Rundles C. Gastrointestinal Manifestations and Complications of Primary Immunodeficiency Disorders. Immunol Allergy Clin North Am 2019;39(1):81-94.

12. Adike A, DiBaise JK. Small Intestinal Bacterial Overgrowth: $\mathrm{Nu}-$ tritional Implications, Diagnosis, and Management. Gastroenterol Clin North Am 2018;47(1):193-208.

13. Edgar D, Ehl S. ESID Registry-Working definitions for clinical diagnosis of PID, 2014.

14. Sachdev AH, Pimentel M. Gastrointestinal bacterial overgrowth: pathogenesis and clinical significance. Ther Adv Chronic Dis 2013;4(5):223-31.

15. Newberry C, Tierney A, Pickett-Blakely O. Lactulose Hydrogen Breath Test Result Is Associated with Age and Gender. Biomed Res Int 2016;2016:1064029.

16. Hermaszewski RA, Webster AD. Primary hypogammaglobulinae mia: a survey of clinical manifestations and complications. Q J Med 1993;86(1):31-42.

17. Hermans PE, Diaz-Buxo JA, Stobo JD. Idiopathic late-onset immunoglobulin deficiency. Clinical observations in 50 patients. Am J Med 1976;61(2):221-37.

18. Atarod L, Raissi A, Aghamohammadi A, et al. A review of gastrointestinal disorders in patients with primary antibody immunodeficiencies during a 10-year period (1990-2000), in children hospital medical center. Iran J Allergy Asthma Immunol 2003;2(2):75-9.

19. Mattsson J, Minaya MT, Monegro M, et al. Outcome of breath tests in adult patients with suspected small intestinal bacterial overgrowth. Gastroenterol Hepatol Bed Bench 2017;10(3):168-72.

20. Rezaie A, Pimentel M, Rao SS. How to Test and Treat Small Intestinal Bacterial Overgrowth: an Evidence-Based Approach. Curr Gastroenterol Rep 2016;18(2):8.

21. Oksenhendler E, Gerard L, Fieschi C, et al. Infections in 252 patients with common variable immunodeficiency. Clin Infect Dis 2008;46(10):1547-54. 